\title{
High Altitude: A Possible Reason for Postoperative Bleeding after Coronary Artery Bypass Graft Operations
}

\author{
Burcin Abud1 ${ }^{*}$, Süreyya Talay², Kemal Karaarslan1, Soysal Turhan1, Celal Selcuk Ünal'3, \\ Emre Kubat ${ }^{3}$ \\ ${ }^{1}$ Cardiovascular Surgery Department, Izmir Tepecik Research and Teaching Hospital, Izmir, Turkey \\ ${ }^{2}$ Cardiovascular Surgery Department, Canakkale State Hospital, Izmir, Turkey \\ ${ }^{3}$ Cardiovascular Surgery Department, Karabük University Research and Teaching Hospital, Karabük, Turkey \\ Email: ${ }^{*}$ burcinabud@hotmail.com
}

Received 17 March 2015; accepted 7 April 2015; published 9 April 2015

Copyright (C) 2015 by authors and Scientific Research Publishing Inc.

This work is licensed under the Creative Commons Attribution International License (CC BY). http://creativecommons.org/licenses/by/4.0/

(c) (i) Open Access

\section{Abstract}

Background: This study evaluated the possible hypothesis that high altitude might be a reason of excessive postoperative drainage after coronary artery bypass graft operations (CABG). Methods: 212 patients were operated by the same surgical team and included to the study with retrospective data scan on postoperative bleeding after elective CABG with cardiopulmonary bypass (CPB). Study was derived from two different altitude centers: Group A patients $(n=107)$ from the center at an altitude of 1985 meters and Group B patients $(n=105)$ from the center in approximately sea level with 150 meters altitude. Multiple preoperative, intraoperative and postoperative characteristics with postoperative bleeding amounts were reviewed and calculated to determine a result through the aim of our study. Results: Preoperative, operative and intraoperative parameters for both groups were almost identical. Statistically significance occurred on intensive care unit treatment period, total hospitalization days, usage of erythrocyte suspension and fresh frozen plasma among postoperative periods on behalf of Group B. Parameters such as CABG numbers, Cross clamp and СРB time were not significant. High altitude patients were considered to be with a higher outcome in preoperative hematocrit (Htc) levels which did not occur in the study group. Htc levels presented a slight elevation for Group A as in the preoperative values but a statistically significance was not calculated. Postoperative bleeding volumes and calculated mean values were statistically significantly different among both groups in days of 1, 2 and 3 . Reoperations for hemorrhage occurred in 9 cases (8.4\%) in Group A and 4 cases (3.8\%) in Group B. Conclusions: We want to attract attention to the relation between high altitude and postoperative bleeding after CABG surgery. But yet uncertain and unidentified physiological and biochemical conditions can be a reason as an uncontrollable perioperative factor in this patients. Operation at high altitude can

${ }^{*}$ Corresponding author.

How to cite this paper: Abud, B., Talay, S., Karaarslan, K., Turhan, S., Ünal, C.S. and Kubat, E. (2015) High Altitude: A Possible Reason for Postoperative Bleeding after Coronary Artery Bypass Graft Operations. World Journal of Cardiovascular Diseases, 5, 79-86. http://dx.doi.org/10.4236/wjcd.2015.54011 


\title{
be a reason for postoperative excessively bleeding.
}

\section{Keywords}

\author{
High Altitude, Postoperative Bleeding, Elective Coronary Artery Bypass Graft Surgery
}

\section{Introduction}

Depending on the criteria of American College of Cardiology and American Heart Association (ACC/AHA), certain degrees of coronary artery atherosclerosis indicate coronary artery bypass grafting (CABG) surgery. Despite the enhanced quality and technique of surgery, postoperative morbidity and mortality still remain a challenging topic of daily practice.

Surgery is performed by various techniques such as CABG with or without cardiopulmonary bypass (CPB), robotic surgery, port access surgery, minimally invasive CABG via thoracotomy, hybrid procedures with concordant stent implantations, intramyocardial laser or stem cell implantations. Prior to operation, EuroScore is a frequently administered method of risk evaluation among this group of patients. Risk factors include emergent surgery, female gender, re-operation, preoperative impair of left ventricular ejection fraction (LVEF), congestive heart failure, existence of severe mitral valve insufficiency, co-existing cerebral disease, chronic obstructive pulmonary disease and renal failure [1].

Despite the developments of cardiac surgery, postoperative complications are presented in every organ system of human body. Mainly, postoperative bleeding, pulmonary dysfunction, mental status deteriorations with a wide variety of neurological sequels, acute myocardial infarction and acute renal failure are early sources of complications [2]-[4].

Postoperative bleeding is a major situation affecting on case mortality and morbidity. The cause of post-cardiac surgery bleeding can be either surgical or nonsurgical [5]-[7]. Early postoperative bleeding with mediastinal and thoracal drainage arises as a result of a multiple number of reasons. Insufficient surgical hemostasis is the most frequent reason compared to other bleeding components such as coagulopathy with preoperative and/or postoperative hematological cellular dysfunction including platelets, perioperative medications, hypertension, massive transfusions of blood and blood products or crystalloids and multiorgan dysfunction [1] [8]-[10]. Reexploration after cardiac surgery is the most feared bleeding-related complication, as it is associated with significant mortality and morbidity [11] [12]. Hence, the effort to obtain safe and effective hemostatic condition is important and can improve patient outcome.

In between these well known and clearly demonstrated bleeding reasons, altitude is an unapproached concern. High altitude is with a several diversifications and physiological adaptations of organ systems. For example, a higher hematocrit level and hypoxia are mostly shared knowledge of this condition. Nevertheless, influences and effects of high altitude on postoperative bleeding are uncertain.

In this present study, we examined the altitude difference as a free determinant of postoperative bleeding in two homogenized groups of patients from two cardiac surgery centers of different altitudes with a maximum effort of eliminating any possible perioperative differentiating components which might change drainage patterns.

\section{Material and Method}

212 patients, which there operated by the same cardiovascular surgical team (experienced cardiovascular surgical team), included to the study with retrospective data scan on postoperative bleeding after elective CABG with CPB. Study derived from two different altitude centers: Group A patients $(n=107)$ from the center at an altitude of 1985 meters and Group B patients $(n=105)$ from the center in approximately sea level with 150 meters altitude. Exclusion criteria from the study are summarized in Table 1 for both groups.

Following at least a week of Aspirin cessation, routine preoperative evaluation performed with physical examination, routine blood tests with cardiac panel, coagulation parameters including prothrombine time (PT) and activated partial thromboplastin time (aPTT), thorax X-Ray, transthoracic echocardiograms and pulmonary evaluation. Transthoracic echocardiograms presented detailed data about cardiac morphology. Artery blood 
Table 1. Exclusion criteria.
1) Patient age lower than 40 and higher than 70
2) Existence of preoperative medically treated COPD or renal disease
3) Existence of preoperative hematological dysfunction
4) Preoperative warfarine and/or fibrinolytic agent treatment
5) Acute MI with emergent surgery
6) Re-operations for CABG
7) Concomitant valve and/or vascular surgery
8) Existence of preoperative atrial fibrillation
9) Intraoperative or early postoperative deaths
10) IABP implantations
11) Beating heart CABG operations
12) LVEF below $35 \%$

COPD = Chronic obstructive pulmonary disease, $\mathrm{MI}=$ Myocardial infarction, $\mathrm{IABP}=$ Intraaortic balloon pump, CABG = Coronary artery bypass graft, LVEF = Left ventricule ejection fraction.

gases were examined in all patients via partial pressure of $\mathrm{O}_{2}$ and $\mathrm{CO}_{2}$. Spirometer tests implied information of preoperative pulmonary functions. Patient age, sex, smoking history and diabetes mellitus incidence were also recorded. Preoperative data approved that two groups were in a homogeneous distribution for medical history and demographical condition as close as possible.

All patients obtained the same premedication with Propofol 2 - $3 \mathrm{mg} / \mathrm{kg}$, Fentanyl Citrate 10 - 15 microgr $/ \mathrm{kg}$ and Pancuronium bromid $0.1 \mathrm{mg} / \mathrm{kg}$. Following endotracheal intubation, anesthesia maintenance dose included Propofol 2 - 5 mg/kg/h and Fentanyl Citrate 5 - 10 microgr $/ \mathrm{kg} / \mathrm{h}$ intravenously (IV). Inhaled anesthesia continued with Sevoflurane.

In Group A and in Group B, coronary artery bypass grafts performed with left intermammarian artery (LIMA) and great sapheneous vein (VSM) only. Other grafts such as radial artery or right intermammarian artery were not used for any case.

Initial activated clotting time (ACT) calculated in both groups. With median sternotomy, LIMA and VSM harvesting were followed by heparin injection with 350 units/per kg to reach the activated clotting time (ACT) for over 400 seconds. Pericardiectomy and aorta-caval cannulation performed. Within 30 centigrade degrees of body core temperature, cross clamp applied aorta alone. Cardiopulmonary bypass was accomplished with centrifugal pump (Bio-Pump BPX-80, Medtronic, Medtronic. Inc., Minneapolis, USA) and membrane oxygenator (Sorin-Biomedica, Dideco, d 708 simplex II, Italy). Cardioplegia administration performed in three phases; 500 $\mathrm{ml}$ of normothermic blood cardioplegia, cold cardioplegia in $10 \mathrm{ml} /$ per $\mathrm{kg}$ in $40 \mathrm{mmHg}$ pressure and $500 \mathrm{ml}$ of hot shot before removal of the cross clamp. Additive cardioplegia doses by cold cardioplegia applied in every 20 minutes for both groups. Coronary anastomoses in total cardiac arrest were followed by the removal of cross clamp. Heparin antagonization reached with Protamine sulphate administration with a 1:1 dose basis. Proximal anastomoses performed with an aortic side-biting clamp. Following the detailed hemostasis maneuvers, surgical drains to mediastinum and thorax when necessary were replaced and sternum closed. Closure ACT levels calculated and confirmed with equality to initial values before leaving the operation room. Intraoperative blood transfusions decisions primarily aimed to reach for the preoperative values when necessary. Patients transported to cardiovascular surgery intensive care unit (ICU). This surgical fashion was applied for both groups similarly.

At this stage, CABG types and numbers, pleural openings, duration of CPB and aortic cross clamp (ACC) were reviewed to compare in both groups. Intensive care unit and hospitalization days were also scanned as a comparative study data. Initial postoperative ICU blood tests recorded as complete blood count (CBC), coagulation parameters by PT and aPTT, electrolytes, renal and liver function tests for both groups.

Postoperative bleeding volume was recorded on a daily basis from all surgical drains until the removal of 
these instruments. We recorded the postoperative data of usage of blood and blood products as well. Postoperative hemostasis maneuvers as also in medications were administered by IV Protamine sulphate and/or Tranexamic acid to reach an equal level of ACT with the preoperative measurements.

\section{Statistics}

All the data obtained in this study were analyzed using the Statistical Package for the Social Sciences for Windows (SPSS Inc., Chicago, Illinois, USA) version 16.0. Data were expressed median (inter-quartile range). For comparisons between the two groups, Chi square test was used for the nominal data, while numerical data were compared using Mann-Whitney U test. A p value less than $0.05(\mathrm{p}<0.05)$ was considered statistically significant.

\section{Results}

In preoperative and demographic data, we did not observe a statistically significant difference on any parameter (Table 2). High altitude patients in Group A are considered to be with a higher preoperative outcome in Hematocrit (Htc) levels which did not occur in the study group. In our opinion, there are two different explanations for this situation. Smoking habits of patients enhance their Htc levels in Group B of lower altitude. Secondly, some of patients in Group A were not residents of this high altitude.

All patients underwent elective CABG operation with CPB. Intraoperative parameters are depicted in Table 3. ACT levels measured twice for each patient; prior to sternotomy and prior to the closure of sternum. Differences between these two results were treated with additive doses of protamine sulfate to ensure the heparin antagonization. Thus, during the transportation to the ICU, patients were equalized for postoperative bleeding by a basal ACT levels. A similar surgical strategy was applied on Htc levels. Following the disconnection of CPB circuit, pre-bypass values for Htc levels were achieved by erythrocyte transfusions for an uneventful early postoperative period as a surgical routine procedure. Short durations of ACC and CPB time were recorded mostly by 1 or 2 vessel CABG cases. Preoperative operative and intraoperative parameters for both groups were almost identical (Table 2 and Table 3).

ICU data consisted of primarily postoperative bleeding and bleeding related parameters. Re-operations for massive bleeding or obvious clinical features of cardiac tamponade were also reviewed in this study. ICU parameters with postoperative data were shown in Table 4.

Table 2. Preoperative and demographic data.

\begin{tabular}{cccc}
\hline Preoperative data & Group A & Group B & p value \\
\hline Age & $59.3(42-70)$ & $61.2(40-70)$ & NS \\
Sex (male) (\%) & 70 & 65 & NS \\
LVEF (\%) & $54.1(35-70)$ & $56.4(35-70)$ & NS \\
DM & 22.4 & 28.1 & NS \\
HT & 46.3 & 39.1 & NS \\
Smoking (years) & 19.4 & 21.7 & NS \\
Hematocrit (\%) & $39.1(31-48)$ & $38.9(28-45)$ & NS \\
Platelet (1000/uL) & $256.7(221-457)$ & $240.2(234-502)$ & NS \\
PT (sec) & $11.9(10.2-15.8)$ & $11.7(11.1-14.9)$ & NS \\
aPTT (sec) & $31.3(22.2-37.8)$ & $29.9(21.9-36.7)$ & NS \\
EuroScore I & $2.7(2.1-4.3)$ & $2.4(2-3.9)$ & NS \\
Total (n) & 107 & 105 & - \\
\hline
\end{tabular}

LVEF = Left ventricle ejection fraction, $\mathrm{DM}=$ Diabetes mellitus, $\mathrm{HT}=$ Hypertension, $\mathrm{PT}=$ Prothrombin time, aPTT $=$ Activated partial thromboplastin time. 
Table 3. Comparison of intraoperative data.

\begin{tabular}{lccc}
\hline \multicolumn{1}{c}{ Intraoperative data } & Group A & Group B & p value \\
\hline Operation time (min) & $210.2(165-280)$ & $195.2(190-330)$ & NS \\
Preoperative ACT (sec) & $108.6(103-230)$ & $98.7(87-210)$ & NS \\
Postoperative ACT (sec) & $110.9(90-120)$ & $103.1(90-110)$ & NS \\
ACC (min) & $82.8(25-130)$ & $86.6(30-165)$ & NS \\
CPB (min) & $138.2(45-200)$ & $120.6(40-330)$ & NS \\
Pleurotomy & & & \\
Unilateral (\%) & 69.4 & 73.9 & NS \\
Bilateral (\%) & 9.7 & 6.2 & NS \\
Number of grafts & $3.2(1-5)$ & $3.3(1-5)$ & NS \\
Total (n) & 107 & 105 & - \\
\hline
\end{tabular}

ACT $=$ Activated clotting time, ACC = Aortic cross clamp, CPB = Cardiopulmonary bypass.

Table 4. Comparison of postoperative data.

\begin{tabular}{lccc}
\hline \multicolumn{1}{c}{ Postoperative data } & Group A & Group B & p value \\
\hline ICU stay (day) & $7.24(3-13)$ & $3.9(2-11)$ & $\mathrm{p}=0.002$ \\
Hospitalization (day) & $16.3(9-28)$ & $8.2(5-24)$ & $\mathrm{p}=0.001$ \\
Early Postoperative & & & \\
Hematocrit (\%) & $29.2(17-35)$ & $27.6(20-45)$ & NS \\
Platelet (1000 u/L) & $101.1(65-187)$ & $109.3(42-203)$ & NS \\
PT (sec) & $14.6(11.6-21.5)$ & $14.3(12.1-23.2)$ & NS \\
aPTT (sec) & $36.2(25.7-46.8)$ & $35.6(28.3-44.2)$ & NS \\
Usage of b \& bp & & $1.3(0-7)$ & $\mathrm{p}=0.0004$ \\
E.S (package) & $4.2(0-8)$ & $1.7(0-6)$ & $\mathrm{p}=0.002$ \\
FFP (package) & $3.3(0-11)$ & $6(5.71 \%)$ & $\mathrm{NS}$ \\
Death & $5(4.67 \%)$ & 105 & - \\
Total (n) & 107 & & \\
\hline
\end{tabular}

ICU = Intensive care unit, PT = Prothrombin time, aPTT = Activated partial thromboplastin time, b\&bp: Blood and blood product, E.S = Erythrocyte suspension, FFP = Fresh frozen plasma.

Statistically significance occurred in the comparative data on ICU period $(p=0.002)$, total hospitalization days $(p=0.001)$, usage of Erythrocyte suspension (ES) $(p=0.0004)$ and Fresh frozen plasma (FFP) $(p=0.002)$ in postoperative period. Other parameters were not statistically significant $(\mathrm{p}>0.05)$. Hematocrit levels presented a slight elevation for Group B as in the preoperative values but a statistically significance was not calculated (Table 4).

Protocol of b \& bp usage were indicated for erythrocyte suspension transfusions with Htc levels under 25 and FFP transfusions with Platelet levels under $80.000 \mathrm{u} / \mathrm{L}$. Platelet suspensions were technically not possible to achieve for both hospitals because of the absence of plasmapheresis units. Depending on this fact, we utilized only erythrocyte suspensions and FFP for every patient when necessary.

Postoperative bleedings were recorded on a daily basis from all drains of mediastinum and thorax. Table 5 consists of postoperative bleeding volumes until the removal of drainage instruments in both groups. Postoperative bleeding volumes and calculated mean values were statistically significantly different among both groups in 
Table 5. Postoperative daily bleeding volumes.

\begin{tabular}{cccc}
\hline Postoperative Day & $\begin{array}{c}\text { Group A } \\
\text { Bleeding Volume (ml) }\end{array}$ & $\begin{array}{c}\text { Group B } \\
\text { Bleeding Volume (ml) }\end{array}$ & p Value \\
\hline Day 1 & $660 \pm 160.5$ & $340 \pm 230.3$ & $\mathrm{p}=0.001$ \\
Day 2 & $225 \pm 260.2$ & $105 \pm 160.1$ & $\mathrm{p}=0.005$ \\
Day 3 & $162 \pm 170.3$ & $50 \pm 10.1$ & $\mathrm{p}=0.003$ \\
Day 4 & $50 \pm 30.4$ & $50 \pm 11.9$ & $\mathrm{NS}$ \\
\hline
\end{tabular}

days of 1,2 and 3 . For the postoperative day of 4 , bleeding volumes reached to a similar level in both groups probably depending on the clinical decisions to remove drainage instruments from the patients.

Re-exploration for hemorrhage following ICU indications was founded on two main situations; sudden massive or excessive bleeding in a stable patient follow-up and certain volumes of hourly drainage. As a clinical protocol; over $500 \mathrm{~mL}$ drainage for the first hour and/or additive $300 \mathrm{~mL}$ bleeding for the following hours constituted a decision for a re-operation. In our study, reoperations for hemorrhage occurred in 9 cases (8.4\%) in Group A and 4 cases (3.8\%) in Group B. None of re-operations were resulted with death in any group.

\section{Discussion}

Postoperative excessive surgical bleeding is a main reason for morbidity and mortality after CABG cases [1]. Hemorrhage causes are mainly divided into two different circumstances: Surgical bleeding and non-surgical bleeding. Clearly, surgical incisions and insufficient suture lines are with excessive bleeding. On the other hand, deterioration effects of CPB on hematologic status [2]-[4], perioperative medications such as aspirin and heparin, postoperative multi-organ dysfunctions and severe hypertension present a tendency for postoperative hemorrhage.

Surgical bleeding may occur as a result of leakage from the anastomotic sites, insufficient hemostatic maneuvers, coronary graft side branches, surgical damage of cardiac and/or major vascular structure and surgical instrument applications. Unsworth-White et al. [8] report a rate of 3.8\% on overall resternotomy for cardiac operations which is dramatically acceptable when compared to resternotomy rates of early attempts of CPB applications with a rate of $15 \%$. They reported that their resternotomy for postoperative hemorrhage cases were more likely in three times for valve repairs than CABG cases. Essential criteria for reexplorations may vary between centers. Hence, a similarity observed in this matter with our study. In our study, reoperations for hemorrhage occurred in 9 cases (8.4\%) in Group A and 4 cases (3.8\%) in Group B. Unsworth-White et al. advocate the Parsonette score system to be useful in predicting postoperative bleeding among their study group which we did not apply in our cases. On the contrary, they reported 5 deaths in their reexploration group that we observed none. Generally, re-exploration prolongs the ICU and total hospitalization periods. Similar results were presented by Dacey et al. [13] among their series of 305 CABG patients they reported reexplorations in a rate of 3.6\% which is significantly lower than our parameters in Group A. Besides, they also reported as in Unsworth-White study, 3 times higher mortality in the re-operative patients with a rate of $9.5 \%$. They report older age, smaller body surface, prolonged CPB and number of distal anastomoses to be associated with increased bleeding risks.

Nonsurgical bleeding after CABG is associated with severe inflammatory response to CPB circuit, prolonged operation variables such as aortic cross clamp time, elevated blood cell damage with disruption of hemostatic inner-mechanisms, especially liver dysfunction with end organ damage, perioperative anti-platelet agent administrations and postoperative severe hypertension [2]-[4] [9] [14]. Intraoperative data of ACT levels, ACC and CPB time values may affect postoperative bleeding directly by obvious mechanisms of coagulopathy. Hence, elimination of the difference in these parameters clearly homogenizes the aspect of ICU bleeding volumes for both groups.

There have been many publications and studies examining these situations which are mentioned above. Nevertheless, the topic of high altitude influence on postoperative bleeding was not possible to detect in any previous medical literature. High altitude is associated with several effects on human organ systems [15] [16]. As a principle the percentage saturation of hemoglobin in red cells with oxygen determines the usable content of oxygen for human cells. Altitude of approximately 2000 meters is an accepted threshold of saturation. After this 
altitude the oxyhemoglobin levels tend to decrease. Physiological adaptations occur in this level within two different aspect; immediate and longer term differentiations. Immediate physiological adaptations to high altitude consist of hyperventilation, fluid loss, heart rate elevations and slightly lowered LV ejection function. On the other hand longer term effects may include reduced glucose utilizations with lower lactate production, compensatory alkali loss by urine output, significant increase in hematocrit and red blood cell levels defined as polycythemia, myoglobin and mitochondria concentration increase, hypoxic pulmonary vasoconstriction, ventricular and interventricular septal hypertrophy, decrease in plasma volume, increased volume of capillaries of skeletal muscle tissue and increase in aerobic enzyme concentration. Lower levels of partial pressure of oxygen at high altitude atmosphere due to hyperventilation may result with hypoxemia which is a condition that triggers carotid bodies. This cascade results hyperventilation in patients. Thus, hyperventilation also causes respiratory alkalosis as a constant metabolic status [15] [16]. As a conclusion, alkalosis results by a compensatory mechanism of increase bicarbonate exertion. From an aspect of postoperative bleeding this single entity requires more investigation which may pass beyond our primary study aim. On the other hand, increased volume of capillaries of skeletal muscle tissue may also lead to the development of excessive bleeding from incised surgical tissue. Nevertheless, physiological adaptation that is mainly affected by high altitude may alter hemostatic cascades among our patients.

\section{Conclusion}

Surgical bleeding after elective CABG operations is definitely multifactorial. Controllable determinatives such as surgical hemostasis, postoperative hypertension, heparin antagonization and platelet dysfunctions are evitable and manageable. In this study, we want to attract attention to the relation between high altitude and postoperative bleeding after CABG surgery. Although ours is a hypothesis with no definitive evidence, uncertain and unidentified physiological and biochemical conditions can be a reason as an uncontrollable perioperative factor in these patients. In our opinion, operation at high altitude can be a reason for postoperative excessively bleeding.

\section{Study Limitations}

Given the smaller patient population size and its retrospective nature, our study may have insufficient power to stable definitive consequences on this postoperative bleeding topic.

\section{Competing Interests}

The authors declare that they have no competing interests.

\section{Authors' Contributions}

BA, ST designed the study. BA, ST, KK and CSÜ carried out studies searching and performed the eligibility assessments. ST evaluated the qualities of the included studies and carried out data extracting. ST analyzed and interpreted the data. BA, ST drafted the manuscript. BA, ST, EK made critical revision of the manuscript for important intellectual content. All authors read and approved the final manuscript.

\section{References}

[1] Frankel, T.L., Stamou, S.C., Lowery, R.C., Kapetanakis, E.I., Hill, P.C., Haile, E. and Corso, P.J. (2005) Risk Factors for Hemorrhage-Related Reexploration and Blood Transfusion after Conventional versus Coronary Revascularization without Cardiopulmonary Bypass. European Journal Cardio-Thoracic Surgery, 27, 494-500. http://dx.doi.org/10.1016/j.ejcts.2004.11.021

[2] Wans, S., Leclerc, J.L. and Vincent, J.L. (1997) Inflammatory Response to Cardiopulmonary Bypass: Mechanisms Involved and Possible Therapeutic Strategies. Chest, 112, 676-692. http://dx.doi.org/10.1378/chest.112.3.676

[3] Fitzgerald, D.C., Holmes, S.D., St Onge, J.R., Ioanou, C., Martin, L.M. and Ad, N. (2015) Systemic Inflammatory Response during Cardiac Surgery: A Pilot Study. Innovations, 10, 125-132. http://dx.doi.org/10.1097/IMI.0000000000000123

[4] Kirklin, J.K. (1991) Prospects for Understanding and Eliminating the Deleterious Effects of Cardiopulmonary Bypass. The Annals of Thoracic Surgery, 51, 812-813. http://dx.doi.org/10.1016/0003-4975(91)90134-C

[5] Yank, V., Tuohy, C.V., Logan, A.C., Bravata, D.M., Staudenmayer, K., Eisenhut, R., et al. (2011) Systematic Review: 
Benefits and Harms of In-Hospital Use of Recombinant Factor VIIa for Off-Label Indications. Annals of Internal Medicine, 154, 529-540. http://dx.doi.org/10.7326/0003-4819-154-8-201104190-00004

[6] Brown, C., Joshi, B., Faraday, N., Shah, A., Yuh, D., Rade, J.J., et al. (2011) Emergency Cardiac Surgery in Patients with Acute Coronary Syndromes: A Review of the Evidence and Perioperative Implications of Medical and Mechanical Therapeutics. Anesthesia \& Analgesia, 112, 777-799. http://dx.doi.org/10.1213/ANE.0b013e31820e7e4f

[7] Clark, K.B., Kon, N.D., Hammon Jr., J.W., MacGregor, D.A., Kincaid, E.H. and Reichert, M.G. (2013) Factor IX Complex for the Treatment of Severe Bleeding after Cardiac Surgery. Journal of Cardiovascular Pharmacology, 62, 6771. http://dx.doi.org/10.1097/FJC.0b013e318290922f

[8] Uns-Worth-White, M.J., Herriot, A., Valencia, O., Poloniecki, J., Smith, E.E.J., Murday, A.J., Parker, J. and Treasure, T. (1995) Resternotomy for Bleeding after Cardiac Operations: A Marker for Increased Morbidity and Mortality. The Annals of Thoracic Surgery, 59, 664-667. http://dx.doi.org/10.1016/0003-4975(94)00995-3

[9] Biancari, F., Airaksinen, K.E.J. and Lip, G.Y. (2012) Benefits and Risks of Using Clopidogrel before Coronary Artery Bypass Surgery: Systematic Review and Meta-Analysis of Randomized Trials and Observational Studies. The Journal of Thoracic and Cardiovascular Surgery, 143, 665-675. http://dx.doi.org/10.1016/j.jtcvs.2011.01.069

[10] Koster, A. and Schirmer, U. (2010) Re-Evaluation of the Role of Antifibrinolytic Therapy with Lysine Analogs during Cardiac Surgery in the Post Aprotinin Era. Current Opinion in Anesthesiology, 24, 92-97. http://dx.doi.org/10.1097/ACO.0b013e32833ff3eb

[11] Karthik, S., Grayson, A.D., McCarron, E.E., Pullan, D.M. and Desmond, M.J. (2004) Reexploration for Bleeding after Coronary Artery Bypass Surgery: Risk Factors, Outcomes, and the Effect of Time Delay. Annals of Thoracic Surgery, 78, 527-534. http://dx.doi.org/10.1016/j.athoracsur.2004.02.088

[12] Ranucci, M., Bozzetti, G., Ditta, A., Cotza, M., Carboni, G. and Ballotta, A. (2008) Surgical Reexploration after Cardiac Operations: Why a Worse Outcome? Annals of Thoracic Surgery, 86, 1557-1562. http://dx.doi.org/10.1016/j.athoracsur.2008.07.114

[13] Dacey, J.L., Munoz, J.J., Baribeaou, Y.R., Johnson, E.R., Lahey, S.J., Leavitt, B.J., Quinn, R.D., Nugent, W.C., Birkmeyer, J.D. and O’connor, G.T. (1998) Reexploration for Hemorrhage Following Coronary Artery Bypass Grafting. Archives of Surgery, 133, 442-447. http://dx.doi.org/10.1001/archsurg.133.4.442

[14] Gay, W.A. (1990) Aspirin, Blood Loss and Transfusion. Annals of Thoracic Surgery, 50, 345. http://dx.doi.org/10.1016/0003-4975(90)90471-H

[15] Levine, B.D. and Stray-Gundersen, J. (1991) Living High-Training Low: Effect of Moderate-Altitude Acclimatization with Low-Altitude Training on Performance. Journal of Applied Physiology, 83, 102-112.

[16] Gore, C.J. and Hopkins, W.G. (1999) Counterpoint: Possitive Effects of Intermittent Hypoxia on Exercise Performance Are Not Mediated Primarily by Augmented Red Cell Volume. Journal of Applied Physiology, 5, 2055-2057. 